\title{
Staphylococcal Scalded Skin Syndrome
}

\author{
Ergin Çiftçi ${ }^{1}$, Anıl Tapısız' ${ }^{1}$ Tanıl Kendirli², Erdal İnce ${ }^{1}$ \\ ${ }^{1}$ Division of Pediatric Infectious Diseases, Department of Pediatrics, Ankara University School of Medicine, \\ Ankara, Turkey \\ 2 Division of Pediatric Intensive Care, Department of Pediatrics, Ankara University School of Medicine, \\ Ankara, Turkey
}

A 10-month girl was referred to the hospital with complaints of rash, blistering and scaling starting from the neck and spreading all over the body. Although there was scaling and crusting in the skin around the mouth, the mouth mucosa was intact.

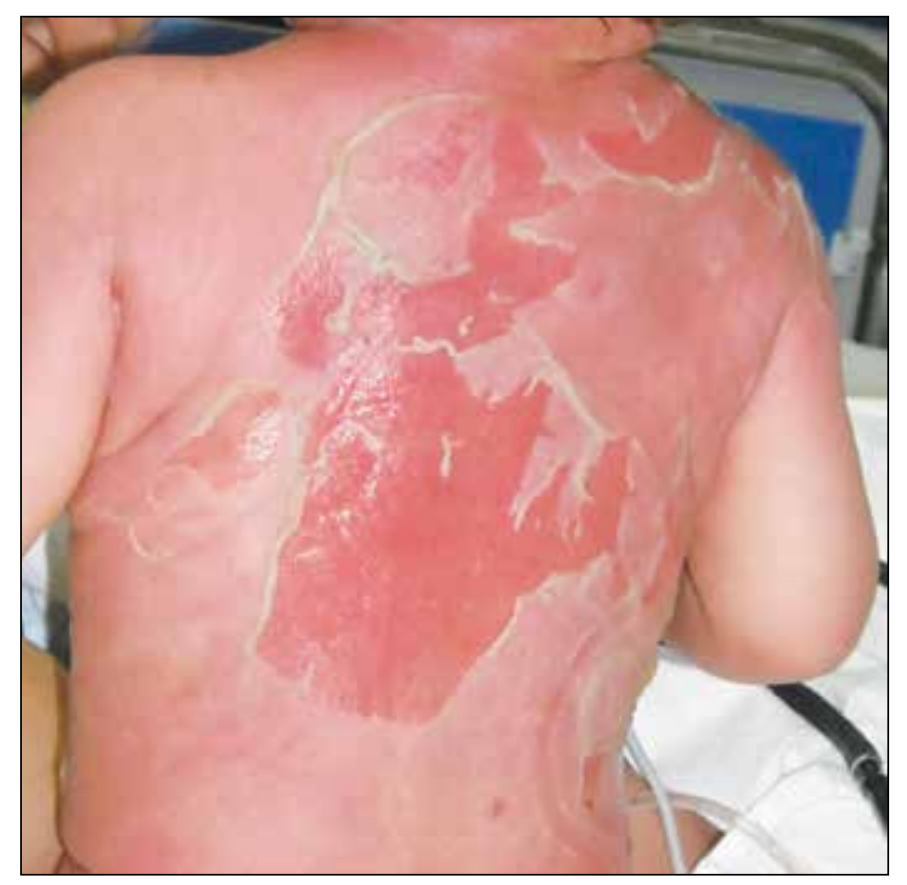

There were erythematous and scaling skin lesions, being more obvious at the back, spreading all over the body. Nikolsky sign was positive.

Staphylococcal Scalded Skin Syndrome (SSSS) was considered in the patient. After taking the cultures, teicoplanin and cefoperazone-sulbactam treatment was initiated. Appropriate intravenous liquid treatment and skin care was initiated. There was no significant growth in the throat, nose, skin lesion swabs and blood cultures of the patient. Skin lesions rapidly faded, dried and crusted. On the fourth day of the treatment, she was discharged with amoxycillin-clavulanic acid treatment. Completing the antibiotic treatment to 10 days, the patient recovered without problems.

SSSS is a severe disease caused by the exfoliative toxins of Staphylococcus aureus. It is more common newborns and children than in adults. The erythema spread wide on the skin and the Nikolsky sign, which ruptures quickly leaving wide lesions is characterized by positive loose bullae. Although skin biopsy makes the diagnosis certain, this is rarely necessary. S. aureus can be detected at any focus, however, unruptured bullae are sterile. Besides appropriate antibiotic treatment for staphylococci, skin care similar to burns and adequate liquid treatment must be used. 